\title{
Characterization of the osteogenic potential of mesenchymal stem cells from human periodontal ligament based on cell surface markers
}

\begin{abstract}
Ruth Alvarez ${ }^{1}$, Hye-Lim Lee ${ }^{1}$, Cun-Yu Wang ${ }^{1}$ and Christine Hong ${ }^{1,2}$
Mesenchymal stem cell (MSC)-mediated therapy has been shown to be clinically effective in regenerating tissue defects. For improved regenerative therapy, it is critical to isolate homogenous populations of MSCs with high capacity to differentiate into appropriate tissues. The utilization of stem cell surface antigens provides a means to identify MSCs from various tissues. However, few surface markers that consistently isolate highly regenerative MSCs have been validated, making it challenging for routine clinical applications and making it all the more imperative to identify reliable surface markers. In this study, we used three surface marker combinations: CD51/CD140 a, CD271, and STRO-1/CD146 for the isolation of homogenous populations of dental mesenchymal stem cells (DMSCs) from heterogeneous periodontal ligament cells (PDLCs). Fluorescence-activated cell sorting analysis revealed that $24 \%$ of PDLCs were $\mathrm{CD} 51^{+} / \mathrm{CD} 140 \alpha^{+}, 0.8 \%$ were $\mathrm{CD} 271^{+}$, and $2.4 \%$ were STRO- $1^{+} / \mathrm{CD} 146^{+}$. Sorted cell populations were further assessed for their multipotent properties by inducing osteogenic and chondrogenic differentiation. All three subsets of isolated DMSCs exhibited differentiation capacity into osteogenic and chondrogenic lineages but with varying degrees. CD271 $1^{+}$DMSCs demonstrated the greatest osteogenic potential with strong induction of osteogenic markers such as DLX5, RUNX2, and BGLAP. Our study provides evidence that surface marker combinations used in this study are sufficient markers for the isolation of DMSCs from PDLCs. These results provide important insight into using specific surface markers for identifying homogenous populations of DMSCs for their improved utilization in regenerative medicine.
\end{abstract}

International Journal of Oral Science (2015) 7, 213-219; doi:10.1038/ijos.2015.42; published 30 October 2015

Keywords: cell surface markers; dental mesenchymal stem cells; periodontal ligament

\section{INTRODUCTION}

Mesenchymal stem cell (MSC)-mediated therapy has been shown to produce promising clinical outcomes in regenerating tissue defects for regenerative medicine and periodontal therapy. ${ }^{1-2}$ Human MSCs are multipotent adult progenitor cells with the capability to self-renew and the potential to differentiate into a variety of cell types including osteoblasts, chondrocytes, and adipocytes. ${ }^{3-4}$ Additional characteristics of MSCs, including their ease of isolation and lack of immunogenicity, make them unique and indispensable tools in tissue engineering and regenerative therapy. ${ }^{5-6}$ While bone marrow is the most widely recognized source of MSCs (bone marrow-derived mesenchymal stem cells (BMSCs)), additional sources for MSCs including craniofacial tissues have been identified. ${ }^{7-9}$ In particular, periodontal ligament (PDL) tissue collected from extracted adult and primary teeth is an easily accessible source of MSCs and such MSCs isolated from the PDL were found to have the ability to regen- erate bone, cementum, and PDL upon in vivo transplantation. ${ }^{10-12}$ As such, the PDL has been identified as a viable and promising source for MSCs in promoting regenerative therapy, especially for craniofacial defects such as periodontal disease. ${ }^{8,11-12}$

The PDL is a dynamic and specialized connective tissue derived from the dental follicle that originates from neural crest cells. ${ }^{13-14}$ PDL tissues contain a heterogeneous population of cells, including fibroblasts, epithelial cells, endothelial cells, cementoblasts, osteoblasts, and neural cells. ${ }^{15}$ Embedded between the cementum and the inner wall of the alveolar bone socket, the PDL's primary functions are to anchor the teeth to the alveolar bone and to provide them with protection against mechanical loads generated by mastication. ${ }^{16}$ In addition to mechanical support, the PDL has many critical biological functions including providing tooth nutrition and regenerating periodontal tissues damaged by inflammatory periodontal disease or mechanical trauma. ${ }^{16}$

${ }^{1}$ Division of Oral Biology and Medicine, School of Dentistry, University of California at Los Angeles, Los Angeles, USA and ${ }^{2}$ Section of Orthodontics, School of Dentistry, University of California at Los Angeles, Los Angeles, USA

Correspondence: DrC Hong, Division of Oral Biology and Medicine, School of Dentistry, University of California at Los Angeles, CHS 63-014, 10833 Le Conte Avenue, Los Angeles CA 90095-1668, USA

E-mail: chong@dentistry.ucla.edu

Professor CY Wang, Division of Oral Biology and Medicine, School of Dentistry, University of California at Los Angeles, CHS 33-030, 10833 Le Conte Avenue, Los Angeles CA 90095-1668, USA

E-mail: cwang@dentistry.ucla.edu

Accepted 17 September 2015 
The role of the PDL is especially important in repair after periodontal disease, which can have acute, chronic, or systemic manifestations, ultimately leading to destruction of periodontal tissue, progressive alveolar bone loss, and eventual tooth loss. ${ }^{17-21}$ This periodontal regeneration is challenging due to the complexity of the PDL attachment apparatus requiring finely orchestrated formation of new cementum, bone, and PDL fibers followed by the insertion of these fibers into the bone and cementum. ${ }^{22}$ Putative periodontal mesenchymal progenitor cells that present properties similar to BMSCs have been characterized from parental PDL cells (PDLCs). ${ }^{7-8,10-11,13,23-25}$ These cells were shown to differentiate into various distinct cell types, such as osteoblasts, fibroblasts, chondrocytes, cementoblasts, adipocytes, and neural-like cells. ${ }^{7-8,10-11,13,23-25}$ They express MSC surface markers such as STRO-1, CD146, STRO-3, CD13, CD29, CD44, CD90, CD105, CD106, and CD166. ${ }^{6,11,17,26}$ In addition, progenitor cells from the PDL express higher levels of scleraxis than MSCs from other tissues including bone marrow and dental pulp, making them a unique population of MSCs. ${ }^{11}$ Dental mesenchymal stem cells (DMSCs) selectively isolated from the PDL with high osteogenic potential are therefore expected to be the best-suited source of progenitor cells for regenerative periodontal therapy. ${ }^{27-28}$ Additional uses of DMSCs from the PDL to improve clinical outcomes in dentistry include regeneration of PDL on the root surface of extracted or avulsed teeth and on titanium implants. ${ }^{29-31}$

Recent studies showed that surface marker combinations, CD51/ CD140 $\alpha$ and CD271/CD90/CD106, isolate highly enriched clonogenic cells from human bone marrow. ${ }^{32-33}$ Similarly, STRO-1/CD146 combination was used to obtain DMSCs from the PDL. ${ }^{23}$ No previous attempts were made to isolate DMSCs from PDLCs using CD51/ CD140 $\alpha$ and CD271. In this study, we used these three cell surface marker combinations to isolate DMSCs from PDLCs and then determine the proportion of PDLCs that are positive for specific surface markers and the magnitude of osteogenic and chondrogenic potentials of these isolated progenitor cells.

\section{MATERIALS AND METHODS}

\section{Cell isolation and culture}

Primary PDL cells (PDLCs) were isolated from the PDL of extracted adult third molars (IRB\#13-000241-CR-00001) as previously described. ${ }^{34}$ PDLCs were cultured in $\alpha$ modified Eagle's medium $(\alpha-$ MEM) (Invitrogen, Carlsbad, CA, USA) containing 20\% fetal bovine serum (FBS), non-essential amino acids, $100 \mathrm{u} \cdot \mathrm{mL}^{-1}$ penicillin, and $100 \mathrm{u} \cdot \mathrm{mL}^{-1}$ streptomycin, in a humidified $5 \% \mathrm{CO}_{2}$ incubator at $37{ }^{\circ} \mathrm{C}$ (all reagents were from Invitrogen, Carlsbad, CA, USA). Media was changed every 2 days, and cells were passaged at $80 \%-90 \%$ confluency. PDLCs used in this study were from passages $4-8$.

\section{Fluorescent-activated cell sorting}

Expression of stem cell surface markers in PDLCs was determined by fluorescent activated cell sorting (FACS) analysis. The cells were detached using trypsin in $0.25 \%$ ethylenediaminetetraacetic acid (EDTA). After neutralization, single-cell suspensions were washed with phosphate-buffered saline (PBS) supplemented with 2\% FBS and $0.01 \% \mathrm{NaN}_{3}$ (FACS buffer). Quantities of $1 \times 10^{6}$ cells were incubated with direct conjugated antibodies for $20 \mathrm{~min}$ on ice in the dark. After washing, fluorescence intensity was measured on FACS Aria II cell sorter (BD Biosciences, San Jose, CA, USA). The following anti-human antibodies were used: phycoerythrin (PE)CD271 (Miltenyi Biotec, Auburn, CA, USA), fluorescein isothiocyanate (FITC)-CD90 (BioLegend, San Diego, CA, USA), allophycocya- nin (APC)-CD106 (BioLegend, San Diego, CA, USA) or dual color combinations APC-STRO-1/PE-CD146 (Both from: BioLegend, San Diego, CA, USA), and PE-CD51 (BioLegend, San Diego, CA, USA)/APC-CD140 $\alpha$ (BD Biosciences, San Jose, CA, USA). PE-IgG was used as a negative control.

\section{Induction of osteogenic differentiation}

Sorted DMSCs were plated at $1 \times 10^{5}$ cells per well into 12 -well plates. To induce osteogenic differentiation into osteogenic lineages, sorted DMSCs were grown in osteogenic induction medium (OIM). OIM contained $\alpha$-MEM (Invitrogen, Carlsbad, CA, USA) supplemented with $10 \%$ FBS (Invitrogen, Carlsbad, CA, USA), $50 \mu \mathrm{g} \cdot \mathrm{mL}^{-1}$ ascorbic acid, $5 \mathrm{mmol} \cdot \mathrm{L}^{-1} \beta$-glycerophosphate, and $100 \mathrm{nmol} \cdot \mathrm{L}^{-1}$ dexamethasone (all from Sigma-Aldrich, St Louis, MO, USA). OIM was changed every 2-3 days. For alkaline phosphatase (ALP) staining, after osteogenic induction for 7 days, cells were fixed with $4 \%$ paraformaldehyde and incubated with a solution of $0.25 \%$ naphthol AS-BI phosphate and $0.75 \%$ Fast blue BB (Sigma-Aldrich, St Louis, MO, USA) dissolved in $0.1 \mathrm{~mol} \cdot \mathrm{L}^{-1}$ tris(hydroxymethyl)aminomethane (Tris) buffer ( $\mathrm{pH}$ 9.3). ALP activity assay was preformed using an ALP kit (Sigma-Aldrich, St Louis, MO, USA) according to the manufacturer's protocol and normalized based on protein concentrations. To detect mineralization potential cells were induced for 2 weeks using OIM, fixed with $4 \%$ paraformaldehyde, and stained with $2 \%$ Alizarin red (Sigma-Aldrich, St Louis, MO, USA). For quantification, Alizarin red was destained with $10 \%$ cetylprydiniumcholoride in $10 \mathrm{mmol} \cdot \mathrm{L}^{-1}$ sodium phosphate for $30 \mathrm{~min}$ at room temperature. Optical absorbance was measured at $562 \mathrm{~nm}$ using a multiplate reader with a standard calcium curve in the same solution. The final calcium level in each group was normalized with the total protein concentrations prepared from a duplicate plate.

\section{Induction of chondrogenic differentiation}

Sorted DMSCs were plated at $1 \times 10^{5}$ cells per well into 12 -well plates. To induce differentiation into chondrogenic lineages, sorted DMSCs were grown in chondrogenic inducing media (CIM) containing $\alpha$ MEM (Invitrogen, Carlsbad, CA, USA) supplemented with 10\% FBS (Invitrogen, Carlsbad, CA, USA), $100 \mathrm{mmol} \cdot \mathrm{L}^{-1}$ sodium pyruvate, $40 \mu \mathrm{g} \cdot \mathrm{mL}^{-1}$ proline, $100 \mathrm{nmol} \cdot \mathrm{L}^{-1}$ dexamethasone, $200 \mu \mathrm{mol} \cdot \mathrm{L}^{-1}$ ascorbic acid (all from Sigma-Aldrich, St Louis, MO, USA), and $10 \mathrm{ng} \cdot \mathrm{mL}^{-1}$ TGF- $\beta 3$ (R\&D systems, Minneapolis, MN, USA). Culture medium was changed every 2-3 days. After 4 weeks of differentiation in vitro, Alcian blue staining (Sigma-Aldrich, St Louis, MO, USA) was performed as previously described. ${ }^{35}$ For quantification, stained Alcian blue was eluted with $6 \mathrm{~mol} \cdot \mathrm{L}^{-1}$ guanidine hydrochloride for 8 hours at room temperature. The optical absorbance was measured at $620 \mathrm{~nm}$ using a microplate reader.

\section{Quantitative reverse transcription-polymerase chain reaction}

The total RNA was isolated from DMSCs using Trizol reagents (Invitrogen, Carlsbad, CA, USA). Two-microgram aliquots of RNAs were used to synthesize cDNAs using random hexamers and reverse transcriptase according to the manufacturer's protocol (Invitrogen, Carlsbad, CA, USA). The real-time polymerase chain reaction (PCR) were performed using the QuantiTect SYBR Green PCR kit (Qiagen, Valencia, CA, USA) and the Icycler IQ Multicolor Real-time PCR Detection System (Bio-Rad, Hercules, CA, USA). The primers for RUNX2 were: forward, 5'-TGGTTACTGTCATGGCGGGTA-3'; reverse, 5'-TCTCAGATCGTTGAACCTTGCTA-3'. The primers for DLX5 were: forward, 5'-GCTCTCAACCCCTACCAGTAT-3'; reverse, 5'-CTTTGGTTTGCCATTCACCATTC-3'. The primers for 
BGLAP were: forward, 5'-AGCAAAGGTGCAGCCTTTGT-3'; reverse, 5'-GCGCCTGGTCTCTTCACT- ${ }^{\prime}$. The primers for PLAP-1 were: forward, 5'-TATTCAACAACCCGGTGAA-3'; reverse, 5'-ACATTCC AAAGTTCCCAAGC-3'. The primers for SOX9 were: forward, 5' CACACAGCTCACTCGACCTT-3'; reverse, 5' -CAAAGGGAATTCT GGTTGGT-3'. The primers for COL2A1 were: forward, 5'CAAGAAGAACTGGTGGAGCA-3'; reverse, 5'-CATAGCTGAAA TGGAAGCCA-3'. The primers for ACAN were: forward, 5' TCGAGGACAGCGAGGCC-3' ; reverse, 5' ${ }^{\prime}$-TCGAGGGTGTAGCG TGTAGAGA-3'.

\section{Statistical analysis}

Unpaired Student's $t$-test was used for statistical analysis $\alpha$ value was set at 0.05 .

\section{RESULTS}

Isolation of DMSCs with surface markers, CD51/CD140 $\alpha$, CD271, STRO-1/CD146 from PDLCs

It has been established that the surface maker combination of STRO-1/ CD146 has successfully isolated DMSCs from dental tissues such as the PDL and apical papilla using FACS analysis ${ }^{23-36}$ Additional markers including CD51/CD140 $\alpha$ and CD271/CD90/CD106 have been suggested for the purpose of identifying multipotent homogenous population of BMSCs and these marker combinations were not previously evaluated in DMSCs. ${ }^{32-33}$ Using two surface marker combinations of CD51/CD140 $\alpha$ and STRO-1/CD146, FACS revealed that $24 \%$ of PDLCs were $\mathrm{CD} 1^{+} / \mathrm{CD} 140 \alpha^{+}$and $2.4 \%$ of PDLCs were STRO- $1^{+} /$ $\mathrm{CD}_{146}{ }^{+}$(Figure 1a and 1c). The isolation of STRO- ${ }^{+} / \mathrm{CD} 146^{+}$ DMSCs is consistent with previous studies, which indicated a $2.6 \%$ isolation of DMSCs from PDL. ${ }^{23}$ Interestingly, we found that the majority of PDLCs (99\%) expressed CD90 (Figure 1d), indicating that CD90 is not sufficiently selective for sorting. On the contrary, FACS using dual markers, CD271 and CD106, revealed that an extremely low proportion of PDLCs were double positive for this combination (Figure 1e). Therefore, we used a single maker, CD271, to isolate DMSCs and found that $0.8 \%$ of PDLCs were CD $271^{+}$, representing the smallest proportion among our combinations (Figure $1 \mathrm{~b}$ ).

\section{Sorted DMSCs exhibited significant differential osteogenic potential}

To evaluate and compare differentiation capacity, isolated $\mathrm{CD}_{51}{ }^{+} /$ $\mathrm{CD} 140 \alpha^{+}, \mathrm{CD} 271^{+}$, and STRO- $1^{+} / \mathrm{CD} 146^{+}$DMSCs were induced to undergo osteogenic differentiation. All three combinations of DMSCs displayed the capacity to differentiate into the osteogenic lineage as demonstrated by ALP staining on the seventh day (Figure 2a). Quantification of ALP activity revealed significant increase of approximately 5-9 fold in all three populations of induced isolated DMSCs compared to non-induced CD $51^{+} / \mathrm{CD} 140 \alpha^{+}$cells (Figure $2 \mathrm{~b}$ ). In addition, all three isolated DMSC groups had formation of mineralized nodules after prolonged treatment with OIM for 14 days as demonstrated by Alizarin red S (ARS) (Figure 2a). Quantification of ARS also showed significant mineralization potential in all induced DMSCs with a 2.5 - to 4 -fold increase compared to non-induced $\mathrm{CD} 51^{+} / \mathrm{CD} 140 \alpha^{+}$ DMSCs.

Using these isolated DMSCs, we further confirmed their osteogenic potential by examining messenger RNA (mRNA) expression of several osteogenic marker genes including DLX5, RUNX2, and BGLAP at different time points: $0,3,7$, and 10 days after osteogenic induction. Consistent with ALP and ARS results, osteogenic marker gene expression was significantly upregulated for all three subsets of isolated DMSCs. In particular, marked increase of DLX5 and RUNX2 expression was found in $\mathrm{CD} 271^{+}$DMSCs at day 7 of induction compared to $\mathrm{CD} 51^{+} / \mathrm{CD} 140 \alpha^{+}$and STRO $-1^{+} / \mathrm{CD} 146^{+}$DMSCs (Figure $3 \mathrm{a}$ and $3 \mathrm{~b}$ ). Significant BGLAP expression induction was found in $\mathrm{CD} 271^{+}$

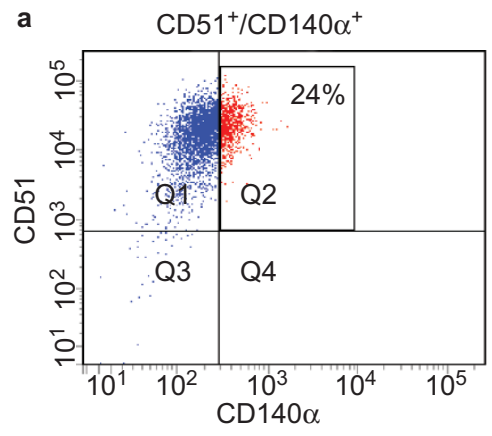

b
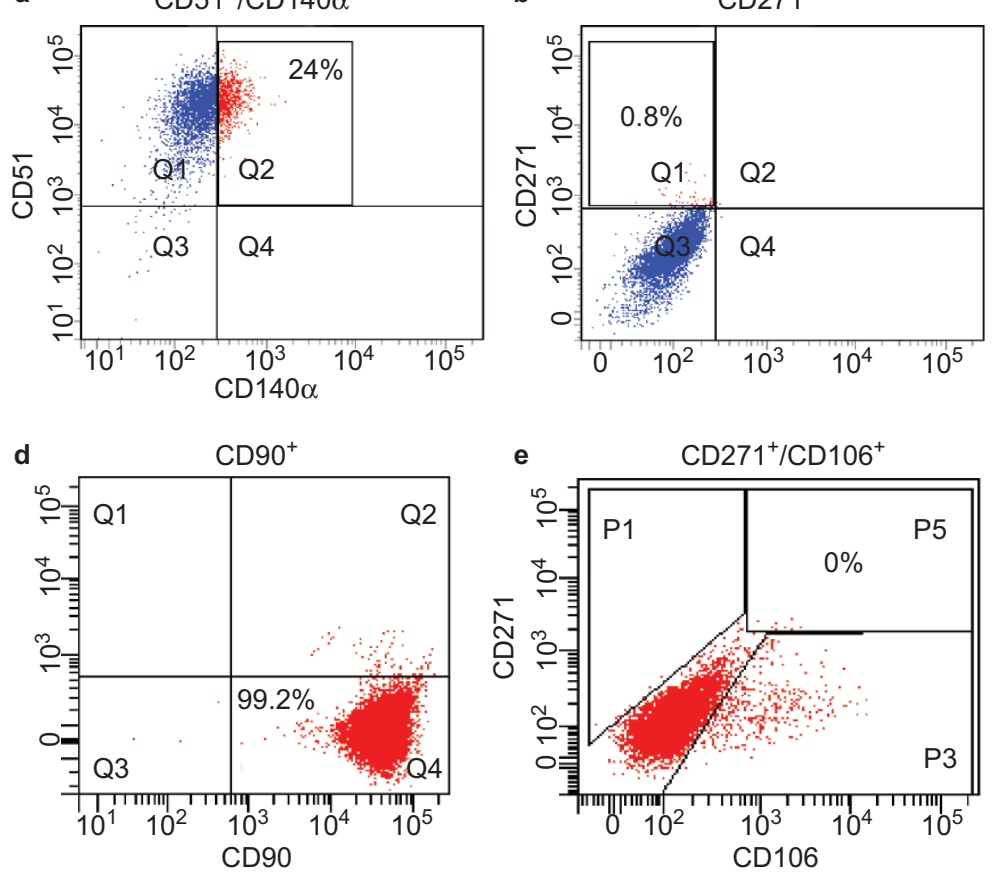

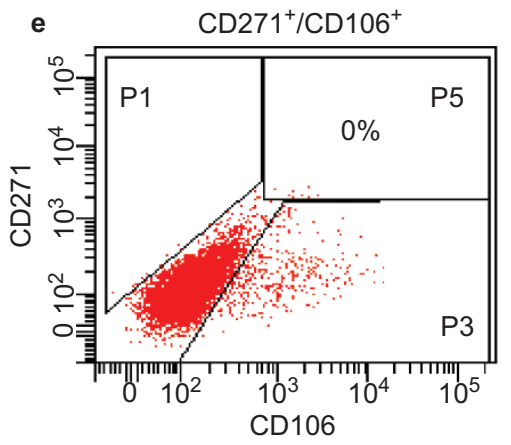

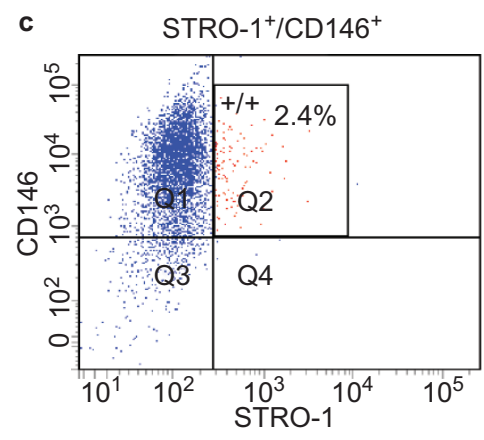

Figure 1 The expression profiles of stem cell surface markers in human primary cells from periodontal ligament tissues determined by FACS. (a) CD51/CD140 $\alpha$, (b) CD271, (c) STRO-1/CD146, (d) CD90, and (e) CD271/CD106. Cells were isolated from the periodontal ligament of adult third molars and stained with antibodies. 
a $\mathrm{DMSC} \quad \mathrm{CD} 51^{+} / \mathrm{CD} 140 \alpha^{+}$ OIM

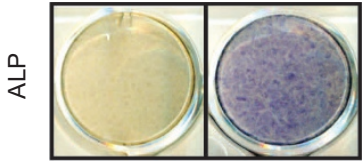

$\stackrel{\infty}{\frac{1}{4}}$
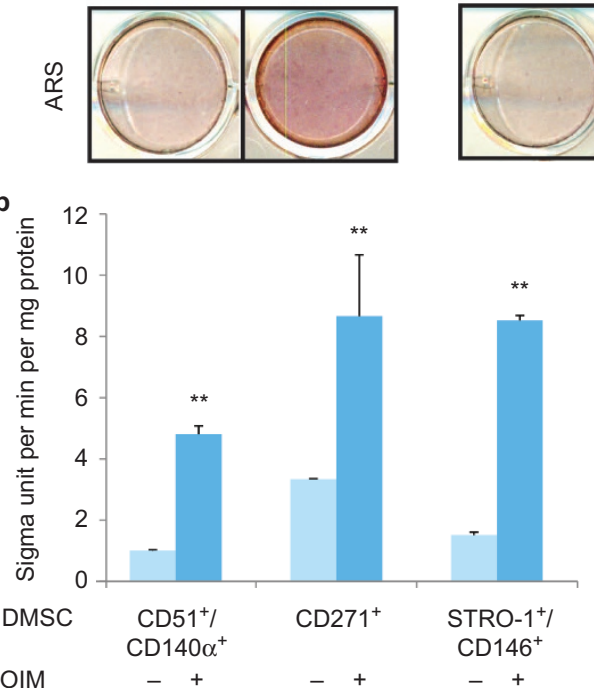

$\mathrm{CD} 271^{+}$
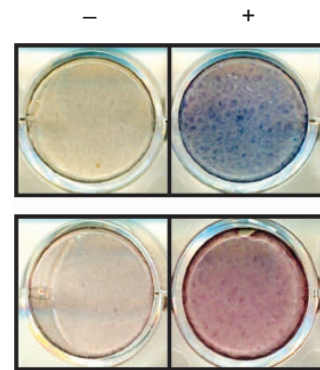

c

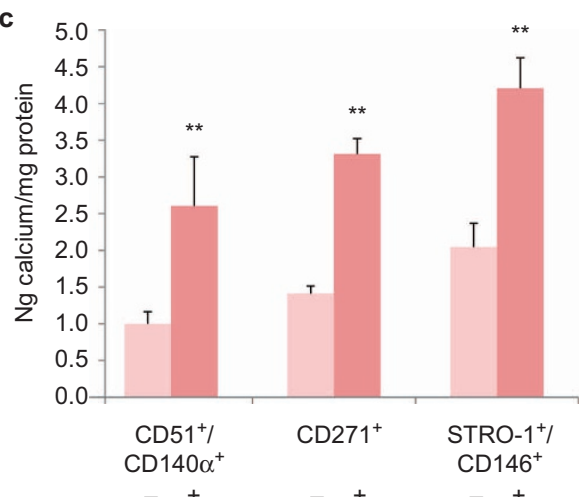

Figure 2 Osteogenic differentiation of isolated CD51 ${ }^{+} / \mathrm{CD} 140 \alpha^{+}, \mathrm{CD} 271^{+}$, and STRO-1/CD146 ${ }^{+}$DMSCs. (a) ALP staining and ARS after these cells were induced to differentiate into odonto/osteogenic lineages for 7 days and 14 days, respectively. (b) Quantification of ALP activity. (c) Quantification of ARS. Values were normalized to non-induced $\mathrm{CD}_{1} 1^{+} / \mathrm{CD} 140 \alpha^{+}$DMSCs. $* P<0.05, * * P<0.001$. ALP, alkaline phosphatase; ARS, Alizarin red S; DMSC, dental mesenchymal stem cell.
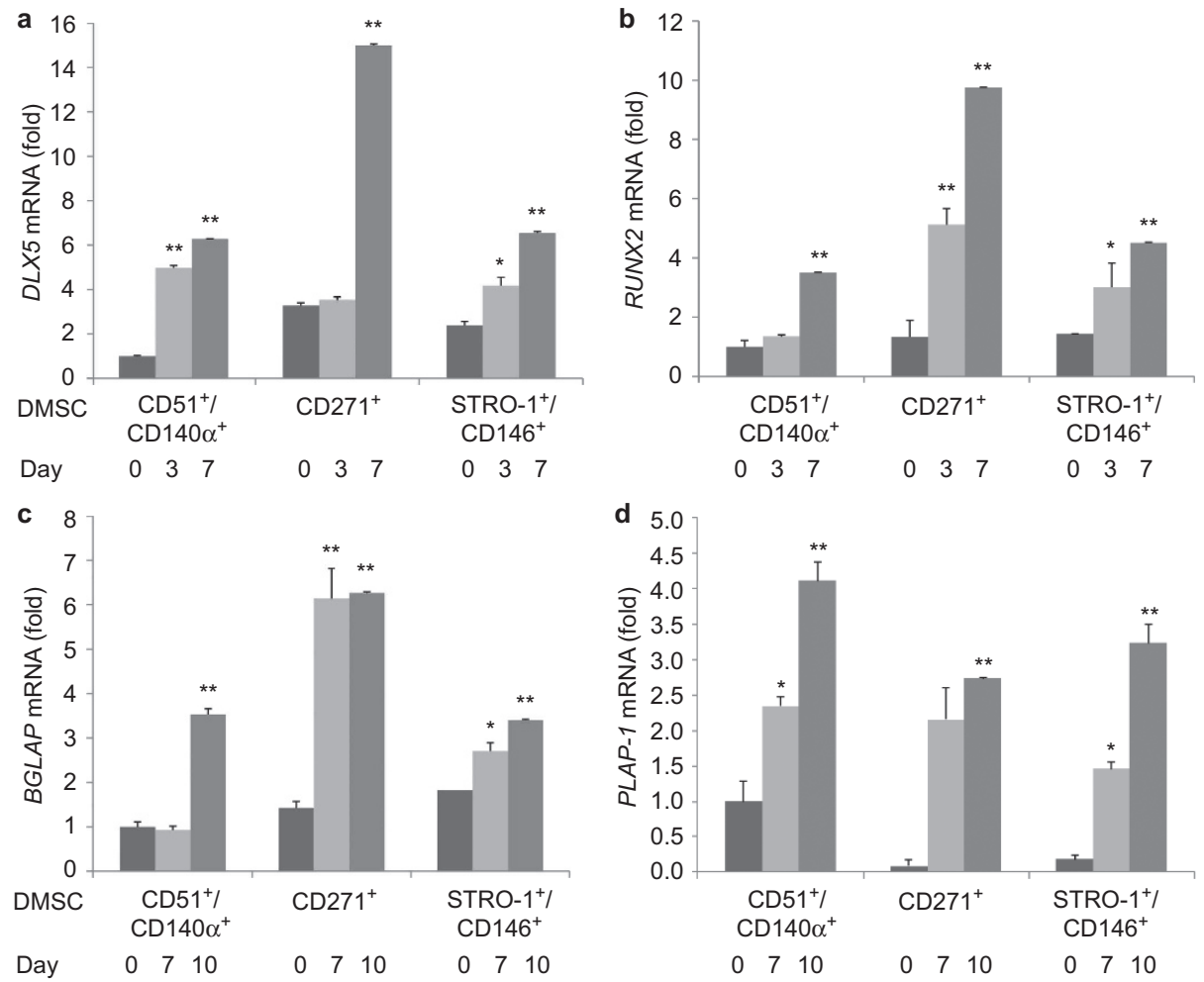

Figure 3 mRNA expression of osteogenic and periodontal genes after $0,3,7$, and 10 days of odonto/osteogenic induction in $C D 51^{+} / C D 140 \alpha^{+}, C D 271^{+}$, and STRO- ${ }^{+} /$CD $146^{+}$DMSCs. (a) DLX5, (b) RUNX2, (c) BGLAP, and (d) PLAP-1. Values were normalized to CD51 ${ }^{+} / \mathrm{CD} 140 \alpha^{+}$DMSCs at day $0 .{ }^{*} P<0.05$, $* * P<0.001$. DMSC, dental mesenchymal stem cell. 
a

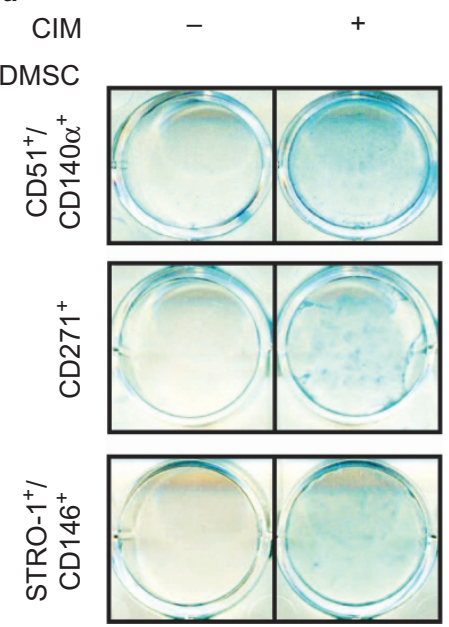

b

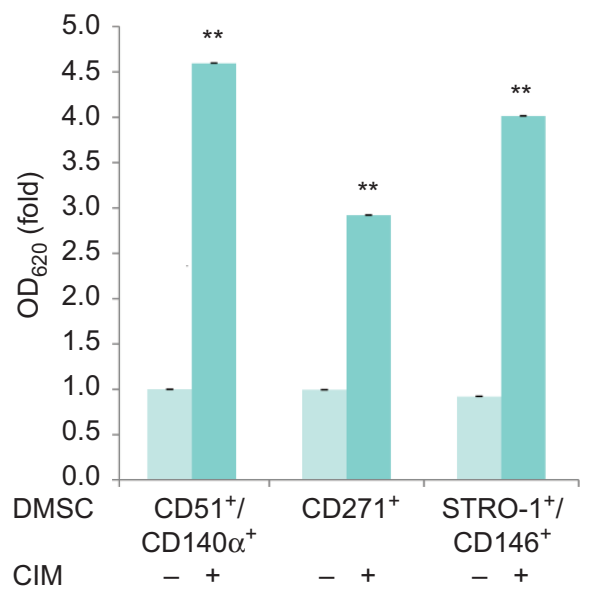

Figure 4 Chondrogenic differentiation of $\mathrm{CD} 51^{+} / \mathrm{CD} 140 \alpha^{+}, \mathrm{CD} 271^{+}$, and STRO- $1^{+} / \mathrm{CD} 146^{+} \mathrm{DMSC}$. (a) Alcian blue staining after cells were induced to differentiate into chondrogenic lineages for 28 days. (b) Quantification of Alcian blue. DMSC, dental mesenchymal stem cell; OD, optical density.

a

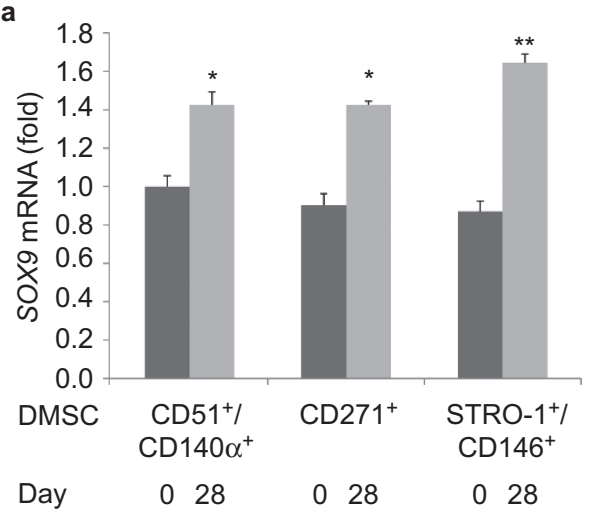

b

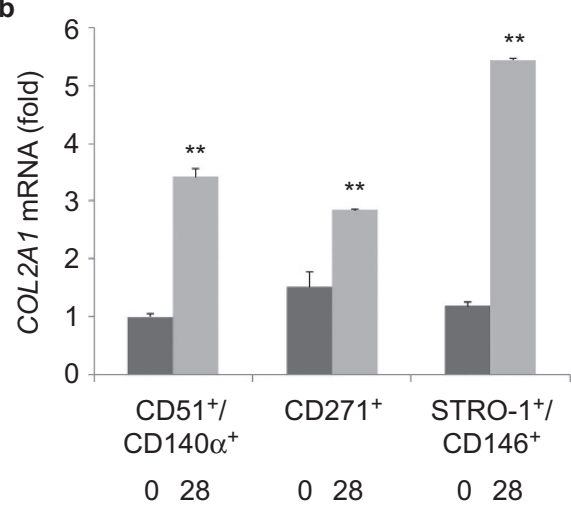

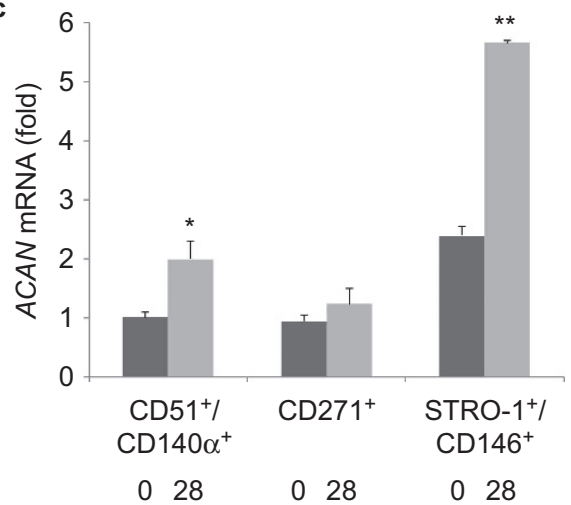

Figure 5 mRNA expression of chondrogenic genes after 0 and 28 days of chondrogenic induction in CD $51^{+} / \mathrm{CD} 140 \alpha^{+}, \mathrm{CD}_{27} 1^{+}$and STRO-1 ${ }^{+} / \mathrm{CD} 146^{+} \mathrm{DMSC}$. (a) SOX9, (b) COL2A1, and (c) ACAN. Values were normalized to CD51 ${ }^{+} / C D 140 \alpha^{+}$DMSCs at day $0 .{ }^{*} P<0.05, * * P<0.001$. DMSC, dental mesenchymal stem cell.

DMSCs at day 7 and 10 of osteogenic induction (Figure 3c). In addition, periodontal marker gene, periodontal ligament-associated protein-1 (PLAP-1), was assessed at 0, 7, 10 days after osteogenic induction. PLAP-1 expression was significantly upregulated in all three subsets of DMSCs (Figure 3d).

Collectively, this data suggested that homogenous populations of DMSCs isolated using FACS with different surface marker combinations have capacity to differentiate into osteogenic lineages.

\section{Isolated DMSCs exhibited significant differential chondrogenic potential}

Furthermore, we evaluated and compared chondrogenic differentiation capacity of isolated DMSCs under chondrogenic conditions. All three isolated DMSC groups showed the presence of glycosaminogylcans as demonstrated by Alcian blue staining after prolonged treatment with CIM for 28 days (Figure 4a). Quantification of Alcian blue staining exhibited a 3- to 4.5-fold increase in chondrogenic differentiation in all three isolated DMSC groups compared to non-induced $\mathrm{CD} 1^{+} / \mathrm{CD} 140 \alpha^{+}$DMSCs (Figure $4 \mathrm{~b}$ ). mRNA expression of several chondrogenic marker genes including SOX9, collagen type 2 alpha 1
(COL2A1), and Aggrecan (ACAN) were significantly induced confirming their chondrogenic potential (Figure $5 \mathrm{a}-5 \mathrm{c}$ ). In particular, all three chondrogenic markers were significantly upregulated in STRO $-1^{+} / \mathrm{CD} 146^{+}$DMSCs (Figure $5 \mathrm{a}-5 \mathrm{c}$ ).

\section{DISCUSSION}

Due to the heterogeneous population of PDLCs, it is critical to selectively isolate highly regenerative MSCs for successful use in clinical settings. We isolated DMSCs from PDL using FACS analysis and three combinations of cell surface markers that have previously been used in successful identification of MSCs from various tissues. ${ }^{23,32-33}$ Our results demonstrated that all three combinations of surface markers, CD51/CD140 $\alpha$, CD271, and STRO-1/CD146 successfully isolated homogenous populations of multipotent progenitor DMSCs from the PDL with varying quantities of the isolated subsets. Although all of these sorted DMSCs showed strong osteogenic potential as evaluated by ALP activity and ARS, the expression of osteogenic marker genes was most significantly upregulated in CD271 ${ }^{+}$DMSCS. The expression of PLAP-1, PDL marker gene, was significantly induced in all of these sorted DMSCs at day 10. Similarly, all three subsets of 
isolated DMSCs exhibited strong chondrogenic differentiation capacity and expression of chondrogenic marker genes was most significantly increased in STRO- $1^{+} / \mathrm{CD} 146^{+}$DMSCs.

Mabuchi et al. first utilized CD271/CD90/CD106 combination of surface markers to selectively isolate a distinct subpopulation of highly potent MSCs in vivo. ${ }^{33}$ Therefore, we initially decided to explore the use of all three markers in PDLCs. Our data showed unreasonably high proportion of PDLCs expressed CD90 (Figure 1d) suggesting that CD90 may cause non-specific isolation of DMSCs. Conversely, FACS analysis revealed that $0 \%$ of PDLCs expressed both CD271 and CD106 (Figure 1e). As such, CD271 alone was used in this study to identify DMSCs from PDLCs. CD271, or p75 neurotrophin receptor ( $\mathrm{p} 75^{\mathrm{NTR}}$ or low affinity nerve growth factor receptor/LNGFR), is a common receptor for all neurotrophins that stimulate neuronal cells to survive and differentiate. ${ }^{37-38}$ Of particular use with BMSCs, it has been demonstrated that CD271 consistently isolates highly multipotent BMSCs and has been described as the most selective marker for the purification and isolation of BMSCs. ${ }^{33,39-43}$

In addition, CD271 has been known for its involvement in epithelial mesenchymal interactions during tooth development. ${ }^{44}$ Progenitor cells from the PDL, which is derived from cranial neural crest-derived ectomesenchyme, are believed to be derived from the neural crest. ${ }^{7-13}$ These neural crest stem cells have been identified in the PDL by immunohistochemistry with CD271 and mRNA expression levels of CD271. ${ }^{7-13}$ However, no previous attempts have been made to isolate progenitor cells using CD271 and FACS analysis.

In our study, we successfully isolated DMSCs from PDL using CD271 but the expression was minimal at just $0.8 \%$ of PDLCs. This low percentage of $\mathrm{CD} 271^{+}$cells is consistent with the results in BMSCs, which reported less than $1 \%$ are CD271 ${ }^{+33,39-43}$ Although CD271 isolated only $0.8 \%$ of DMSCs, CD271 ${ }^{+}$cells had highly significant odonto/osteogenic potential and produced the greatest upregulation of osteogenic marker genes during induction. Alexander et al. previously reported that CD271 is an early surface marker of osteogenic capacity in vitro in human jaw periosteum-derived progenitor cells (JPCs) as CD271 was able to distinguish between mineralizing JPCs and non-mineralizing JPCs using FACS analysis. ${ }^{45}$

STRO-1 and CD146 are MSC surface markers that have been used individually and in combination to identify MSCs from various tissues. ${ }^{11,23,36,46-49}$ STRO-1, a cell membrane single pass type I protein is involved in calcium signaling. ${ }^{9}$ Initially, it was associated with the identification of osteogenic precursors isolated from bone marrow and later described as a promising marker for MSCs. ${ }^{13-46}$ CD146 or the melanoma cell adhesion molecule is a key cell adhesion protein in vascular endothelial cell activity and angiogenesis and is associated with vascular smooth muscle commitment. ${ }^{9,48,50}$ Our study with FACS analysis using the STRO-1/CD146 combination isolated 2.4\% of PDLCs and these sorted STRO- ${ }^{+} / \mathrm{CD} 146^{+}$DMSCs demonstrated high osteogenic potential (Figure 1c), confirming the results of a previous study. ${ }^{23}$ Consistent with the study by Xu et al., the adipogenic potential of STRO $-1^{+} / \mathrm{CD} 146^{+}$DMSCs was insignificant (data not shown), confirming a preferential bias of STRO- $1^{+} / \mathrm{CD} 146^{+}$ DMSCs toward osteogenic and chondrogenic differentiation. ${ }^{23}$

The intermediate filament protein, Nestin, identifies a distinct population of highly multipotent MSCs from mouse bone marrow. ${ }^{32}$ However, because of the intracellular location of Nestin, its utilization to identify BMSCs in vivo would require cell permeabilization preventing the isolation of live cells. ${ }^{32}$ In order to address this shortcoming, Pinho et al. used CD51/CD140 $\alpha$ cell marker combination to isolate a stromal population that is Nestin + and highly enriched in
MSCs in the mouse and human bone marrow. ${ }^{32}$ Clonally expanded $\mathrm{CD} 1^{+} / \mathrm{CD} 140 \alpha^{+}$cells exhibited robust differentiation potential into osteoblastic, adipocytic, and chondrocytic lineages in vivo. ${ }^{32}$

CD51 identifies the integrin vitronectin $\alpha$ chain receptor, important for adhesion and development while CD140 $\alpha$ /platelet-derived growth factor receptor $\alpha$ is a tyrosine kinase receptor expressed on mesenchymal derived cells. ${ }^{51-54}$ In our study, a high proportion $(24 \%)$ of PDLCs were positive for CD51 and CD140 $\alpha$. This large yield of $\mathrm{CD} 1^{+} / \mathrm{CD} 140 \alpha^{+}$DMSCs from the PDL is consistent with high Nestin expression in the majority of adult neural crest stem cells. ${ }^{55-56}$ In addition, these isolated $\mathrm{CD} 51^{+} / \mathrm{CD} 140 \alpha^{+}$DMSCs were successfully induced to undergo differentiation into osteogenic and chondrogenic lineages. Quantification of ALP activity and ARS and expression of osteogenic marker genes exhibited comparably significant osteogenic and chondrogenic potential for $\mathrm{CD} 1^{+} / \mathrm{CD} 140 \alpha^{+}$DMSCs as STRO$1^{+} /$CD $146^{+}$DMSCs.

Our findings not only offer recommendations for isolating MSCs from PDL, but also provide future directions for clinical applications of DMSCs in dentistry and medicine, including periodontal therapy. Periodontal disease is a major cause of tooth loss and a substantial public health concern. ${ }^{19}$ Caused by precipitating factors such as microorganisms and their byproducts, periodontitis is initiated by an inflammatory process that leads to the dissolution of tissue components. ${ }^{17-20}$ The selective isolation of DMSCs from the PDL may offer the possibility of improvements in regenerating the periodontal apparatus that is destroyed by periodontal disease. Additional clinical applications of DMSCs from PDL in dentistry include periodontal defect repair, PDL development for titanium dental implants, and tooth root repair. ${ }^{12,27,57-58}$

In conclusion, our findings demonstrated the successful isolation of distinct subpopulations of DMSCs from human PDL with the use of CD51/CD140 $\alpha$, CD271, and STRO-1/CD146 surface markers and demonstrated their capacity to undergo differentiation into osteogenic and chondrogenic lineages. Each marker yielded a different quantity of isolated mesenchymal progenitor cells with varying magnitude of multi-lineage differentiation potential. As CD51/CD140 $\alpha$ produced isolation of significantly higher proportion of PDLCs than the other two cell surface marker combinations, CD51/CD140 $\alpha$ may be a sufficient marker combination to use with FACS analysis to obtain highly multipotent MSCs from the PDL. Further studies are needed to validate whether these isolated cells may differentiate into functionally different lineages in vivo.

\section{ACKNOWLEDGEMENTS}

This study was supported by National Institute of Dental and Craniofacial Research grant T90DE022734.

1 Horwitz EM, Prockop DJ, Fitzpatrick LA et al. Transplantability and therapeutic effects of bone marrow-derived mesenchymal cells in children with osteogenesis imperfecta. Nat Med 1999; 5(3): 309-313.

2 Yamada $\mathrm{Y}$, Ueda $\mathrm{M}$, Hibi $\mathrm{H}$ et al. A novel approach to periodontal tissue regeneration with mesenchymal stem cells and platelet-rich plasma using tissue engineering technology: a clinical case report. Int J Periodontics Restorative Dent 2006; 26(4): 363-369.

3 Pittenger MF, Mackay AM, Beck SC et al. Multilineage potential of adult human mesenchymal stem cells. Science 1999; 284(5411): 143-147.

4 Wei X, Yang X, Han ZP et al. Mesenchymal stem cells: a new trend for cell therapy. Acta Pharmacol Sin 2013; 34(6): 747-754.

5 Chen FH, Tuan RS. Mesenchymal stem cells in arthritic diseases. Arthritis Res Ther 2008; 10(5): 223.

6 Wada N, Menicanin D, Shi S et al. Immunomodulatory properties of human periodontal ligament stem cells. J Cell Physiol 2009; 219(3): 667-676. 
7 Huang GT, Gronthos S, Shi S. Mesenchymal stem cells derived from dental tissues vs. those from other sources: their biology and role in regenerative medicine. $J$ Dent Res 2009; 88(9): 792-806.

8 Park JC, Kim JM, Jung IH et al. Isolation and characterization of human periodonta ligament (PDL) stem cells (PDLSCs) from the inflamed PDL tissue: in vitro and in vivo evaluations. J Clin Periodontol 2011; 38(8): 721-731.

9 Lv FJ, Tuan RS, Cheung KM et al. Concise review: the surface markers and identity of human mesenchymal stem cells. Stem Cells 2014; 32(6): 1408-1419.

10 Silvério KG, Rodrigues TL, Coletta RD et al. Mesenchymal stem cell properties of periodontal ligament cells from deciduous and permanent teeth. J Periodonto 2010; 81(8): 1207-1215.

11 Seo BM, Miura M, Gronthos S et al. Investigation of multipotent postnatal stem cells from human periodontal ligament. Lancet 2004; 364(9429): 149-155.

12 Liu Y, Zheng Y, Ding G et al. Periodontal ligament stem cell-mediated treatment for periodontitis in miniature swine. Stem Cells 2008; 26(4): 1065-1073.

13 Coura GS, Garcez RC, de Aguiar CB et al. Human periodontal ligament: a niche of neural crest stem cells. J Periodont Res 2008; 43(5): 531-536.

14 Miletich I, Sharpe PT. Neural crest contribution to mammalian tooth formation. Birth Defects Res C Embryo Today 2004; 72(2): 200-212.

15 Lekic $\mathrm{P}$, Rojas J, Birek $\mathrm{C}$ et al. Phenotypic comparison of periodontal ligament cells in vivo and in vitro. J Periodont Res 2001; 36(2): 71-79.

16 Shimono $\mathrm{M}$, Ishikawa $\mathrm{T}$, Ishikawa $\mathrm{H}$ et al. Regulatory mechanisms of periodontal regeneration. Microsc Res Tech 2003; 60(5): 491-502.

17 Nagatomo K, Komaki M, Sekiya I et al. Stem cell properties of human periodontal ligament cells. J Periodont Res 2006; 41(4): 303-310.

18 Reinhardt RA, Payne JB, Maze CA et al. Influence of estrogen and osteopenia/ osteoporosis on clinical periodontitis in postmenopausal women. J Periodontol 1999; 70(8): 823-828.

19 Petersen PE, Ogawa $\mathrm{H}$. The global burden of periodontal disease: towards integration with chronic disease prevention and control. Periodontol 2000 2012; 60(1): 15-39.

$20 \mathrm{Kim} \mathrm{SH}$, Seo BM, Choung PH et al. Adult stem cell therapy for periodontal disease. Int J Stem Cells 2010; 3(1): 16-21.

21 Lindhe J, Lang NP. Clinical periodontology and implant dentistry. 6th ed. Oxford: Wiley-Blackwell, 2015.

22 Bartold PM, Xiao Y, Lyngstaadas SP et al. Principles and applications of cell delivery systems for periodontal regeneration. Periodontol $20002006 ; 41$ : 123-135.

$23 \mathrm{Xu} J$, Wang W, Kapila Y et al. Multiple differentiation capacity of STRO- $1^{+} / \mathrm{CD} 146^{+}$ PDL mesenchymal progenitor cells. Stem Cells Dev 2009; 18(3): 487-496.

24 Bueno C, Ramirez C, Rodríguez-Lozano FJ et al. Human adult periodontal ligamentderived cells integrate and differentiate after implantation into the adult mammalian brain. Cell Transplant 2013; 22(11): 2017-2028.

25 Li X, Gong P, Liao D. In vitro neural/glial differentiation potential of periodonta ligament stem cells. Arch Med Sci 2010; 6(5): 678-685.

26 Trubiani O, Di Primio R, Traini T et al. Morphological and cytofluorimetric analysis of adult mesenchymal stem cells expanded ex vivo from periodontal ligament. Int J Immunopathol Pharmacol 2005; 18(2): 213-221.

27 Iwata T, Yamato M, Zhang Z et al. Validation of human periodontal ligament-derived cells as a reliable source for cytotherapeutic use. J Clin Periodontol 2010; 37(12): 1088-1099.

28 Lin NH, Gronthos S, Bartold PM. Stem cells and future periodontal regeneration. Periodontol 2000 2009; 51: 239-251.

29 Dangaria SJ, Ito $Y$, Luan $X$ et al. Successful periodontal ligament regeneration by periodontal progenitor preseeding on natural tooth root surfaces. Stem Cells Dev 2011; 20(10): 1659-1668.

30 Gault P, Black A, Romette JL et al. Tissue-engineered ligament: implant constructs for tooth replacement. J Clin Periodontol 2010; 37(8): 750-758.

$31 \mathrm{Kim}$ RH, Mehrazarin S, Kang MK. Therapeutic potential of mesenchymal stem cells for oral and systemic diseases. Dent Clin North Am 2012; 56(3): 651-675.

32 Pinho S, Lacombe J, Hanoun M et al. PDGFR $\alpha$ and CD51 mark human Nestin ${ }^{+}$sphereforming mesenchymal stem cells capable of hematopoietic progenitor cell expansion. J Exp Med 2013; 210(7): 1351-1367.

33 Mabuchi Y, Morikawa S, Harada S et al. LNGFR ${ }^{+}$THY $-1^{+}$VCAM- $1^{\text {hi }}$ cells reveal functionally distinct subpopulations in mesenchymal stem cells. Stem Cell Reports 2013; 1(2): 152-165.

34 Fan Z, Yamaza T, Lee JS et al. BCOR regulates mesenchymal stem cell function by epigenetic mechanisms. Nat Cell Biol 2009; 11(8): 1002-1009.

35 Arpornmaeklong P, Brown SE, Wang Z et al. Phenotypic characterization, osteoblastic differentiation, and bone regeneration capacity of human embryonic stem cell-derived mesenchymal stem cells. Stem Cells Dev 2009; 18(7): 955-968.
36 Bakopoulou A, Leyhausen G, Volk J et al. Comparative characterization of STRO- $1^{\text {neg/ }}$ CD $146^{\text {pos }}$ and STRO- $1^{\text {pos }} /$ CD $146^{\text {pos }}$ apical papilla stem cells enriched with flow cytometry. Arch Oral Biol 2013; 58(10): 1556-1568.

37 Tomellini E, Lagadec C, Polakowska R et al. Role of p75 neurotrophin receptor in stem cell biology: more than just a marker. Cell Mol Life Sci 2014; 71(13): 2467-2481.

$38 \mathrm{He}$ XL, Garcia KC. Structure of nerve growth factor complexed with the shared neurotrophin receptor p75. Science 2004; 304(5672): 870-875.

39 Alvarez-Viejo M, Menendez-Menendez Y, Blanco-Gelaz MA et al. LNGFR (CD271) as a marker to identify mesenchymal stem cells from different human sources: umbilical cord blood, Wharton's jelly and bone marrow. Bone Marrow Res 2013; 1(132): 1-6.

40 Kuçi S, Kuçi Z, Kreyenberg H et al. CD271 antigen defines a subset of multipotent stromal cells with immunosuppressive and lymphohematopoietic engraftmentpromoting properties. Haematologica 2010; 95(4): 651-659.

41 Jones EA, Kinsey SE, English A et al. Isolation and characterization of bone marrow multipotential mesenchymal progenitor cells. Arthritis Rheum 2002; 46(12): 33493360.

42 Quirici N, Soligo D, Bossolasco P et al. Isolation of bone marrow mesenchymal stem cells by anti-nerve growth factor receptor antibodies. Exp Hematol 2002; 30(7): 783791.

43 Flores-Torales E, Orozco-Barocio A, Gonzalez-Ramella OR et al. The CD271 expression could be alone for establisher phenotypic marker in bone marrow derived mesenchymal stem cells. Folia Histochem Cytobiol 2010; 48(4): 682-686.

44 Nosrat CA, Fried K, Lindskog S et al. Cellular expression of neurotrophin mRNAs during tooth development. Cell Tissue Res 1997; 290(3): 569-580.

45 Alexander D, Schäfer F, Munz A et al. LNGFR induction during osteogenesis of human jaw periosteum-derived cells. Cell Physiol Biochem 2009; 24(3/4): 283-290.

46 Stewart K, Walsh S, Screen J et al. Further characterization of cells expressing STRO-1 in cultures of adult human bone marrow stromal cells. J Bone Miner Res 1999; 14(8): 1345-1356.

47 Sacchetti B, Funari A, Michienzi S et al. Self-renewing osteoprogenitors in bone marrow sinusoids can organize a hematopoietic microenvironment. Cell 2007; 131(2): 324-336.

48 Espagnolle N, Guilloton F, Deschaseaux F et al. CD146 expression on mesenchymal stem cells is associated with their vascular smooth muscle commitment. J Cell Mol Med 2014; 18(1): 104-114.

49 Shi S, Gronthos S. Perivascular niche of postnatal mesenchymal stem cells in human bone marrow and dental pulp. J Bone Miner Res 2003; 18(4): 696-704.

50 Shih IM. The role of CD146 (Mel-CAM) in biology and pathology. J Pathol 1999; 189(1): 4-11.

51 Chong JJ, Reinecke $\mathrm{H}$, Iwata $\mathrm{M}$ et al. Progenitor cells identified by PDGFR-alpha expression in the developing and diseased human heart. Stem Cells Dev 2013; 22(13): 1932-1943.

52 Hynes RO. Integrins: versatility, modulation, and signaling in cell adhesion. Cell 1992; 69(1): 11-25.

53 Wilder RL. Integrin alpha $V$ beta 3 as a target for treatment of rheumatoid arthritis and related rheumatic diseases. Ann Rheum Dis 2002; 61(Suppl 2): ii96-ii99.

54 Yubero N, Jiménez-Marín A, Barbancho $\mathrm{M}$ et al. Two cDNAs coding for the porcine CD51 ( $\alpha$ v) integrin subunit: cloning, expression analysis, adhesion assays and chromosomal localization. Gene 2011; 481(1): 29-40.

55 Park D, Xiang AP, Mao FF et al. Nestin is required for the proper self-renewal of neural stem cells. Stem Cells 2010; 28(12): 2162-2171.

56 Kaltschmidt B, Kaltschmidt C, Widera D. Adult craniofacial stem cells: sources and relation to the neural crest. Stem Cell Rev 2012; 8(3): 658-671.

57 Lee $\mathrm{CH}$, Hajibandeh J, Suzuki T et al. Three-dimensional printed multiphase scaffolds for regeneration of periodontium complex. Tissue Eng Part A 2014; 20(7/8): 13421351.

58 Lin Y, Gallucci GO, Buser D et al. Bioengineered periodontal tissue formed on titanium dental implants. J Dent Res 2011; 90(2): 251-256.

This work is licensed under a Creative Commons Attribution-

NonCommercial-NoDerivs 4.0 Unported License. The images or other third party material in this article are included in the article's Creative Commons license, unless indicated otherwise in the credit line; if the material is not included under the Creative Commons license, users will need to obtain permission from the license holder to reproduce the material. To view a copy of this license, visit http://creativecommons.org/licenses/ by-nc-nd/4.0/ 